\title{
Post-flambage unilatéral des films minces sur substrat
}

\section{Transitions entre équilibres}

\author{
Guillaume Parry — Jérôme Colin — Christophe Coupeau \\ Alain Cimetière
}

LMP (CNRS - UMR 6630), BP 30179, F-86962 Futuroscope cedex

guillaume.parry@ltpcm.inpg.fr

RÉSUMÉ. Le flambage intervenant sur les zones de décohésion des films minces offre à l'observateur de nombreuses formes différentes, caractérisées par des états d'équilibre se situant en régime post-critique lointain. Des résultats sont présentés issus de la modélisation par éléments finis des équilibres post-flambés d'un film mince délaminé suivant une bande rectiligne et s'appuyant sur un substrat rigide plan. Le contact est sans frottement. Les résultats des calculs sont comparés à ceux obtenus lors d'une étude expérimentale permettant d'observer par microscopie à force atomique les évolutions des différents équilibres sous forme de ride droite, de bulles ou de cordons de téléphone, en faisant varier l'état de contraintes biaxiales dans le film. La nature sous-critique ou sur-critique des transitions est identifiée et les évolutions des longueurs d'onde des structures dans le régime post-critique sont également déterminées. Une cartographie des différents états post-critiques stables est fournie.

ABSTRACT. Various buckling equilibria can be observed on the delaminated areas of thin films. These equilibria are usually under advanced post-buckling. Results of the finite element analysis for the post-buckling equilibria of a thin film delaminated along a rectangular strip are discussed in this paper. Those results are compared with experimental observations performed with the help of an in-situ atomic force microscope coupled to a compression device which allows to vary the biaxial stress state in the film. The transitions from straightsided blisters to bubbles or telephone cord blisters are characterized. Both sub-critical and super-critical transitions are highlighted. A mapping of the various stable post-critical equilibria is given.

MOTS-CLÉS : flambage unilatéral, films minces, calculs par éléments finis, stabilité, analyse post-critique.

KEYWORDS: unilateral buckling, thin films, finite element analysis, stability, bubbles, postcritical analysis.

DOI:10.3166/REMN.16.941-955 @ 2007 Lavoisier, Paris 


\section{Introduction}

L'utilisation des revêtements est un point commun à de nombreuses applications technologiques, parmi lesquelles on peut compter la micro-électronique, les barrières de corrosion et les barrières de protection thermique. Les couches déposées sont soumises à des contraintes de compression très élevées induisant le délaminage et le flambage du film. Ces phénomènes ont étés étudiés depuis des années dans le cadre de la mécanique des solides et de la science des matériaux (Wang et al., 1999; Hutchinson et al., 1992b; Hutchinson, 2001).

Les motifs de flambage sont observés sur les parties délaminées des films, ces dernières ayant été séparées du substrat spontanément à cause des fortes contraintes de compression ou bien suite à des sollicitations mécaniques extérieures. Les surfaces délaminées ont souvent la forme de longues bandes rectangulaires, avec une longueur de l'ordre de plusieurs centaines de fois la largeur ou plus. Les motifs les plus fréquemment observés sont connus sous le nom de ride droite, bulles (il s'agit alors d'un alignement de bulles le long de la bande délaminée) et cordon de téléphone (Gille $e t$ al., 1984; Gioia et al., 1997; Cleymand et al., 2001).

Les équations de Von-Karman pour les plaques élastiques minces en grands déplacements et petites déformations ont été l'outil de base pour les calculs analytiques de flambage. La résolution explicite de ces équations dans le cas de la ride droite fournit son état en régime post-critique (Hutchinson et al., 1992a). Dans le cas des bulles et des cordons de téléphone, les équations de Von Karman ne peuvent pas être résolues analytiquement pour obtenir les modes de flambage naissant ni l'évolution post-critique de ces structures. Des analyses linéaires ont été menées en introduisant des perturbations de forme sur la solution connue de ride droite, fournissant ainsi des résultats sur le flambage naissant des structures de bulles ou de cordon de téléphone (Audoly, 1999; George et al., 2002). Ces deux modes sont des modes de flambage secondaire, l'état de ride droite étant le flambage primaire de référence. Récemment, l'analyse post-critique basée sur la description par éléments finis de ces structures de flambage a permis une meilleure compréhension des transitions entre ces différents équilibres (Parry et al., 2004; Parry et al., 2005).

Nous montrons ici comment la modélisation par éléments finis des bandes délaminées dans le cadre des calculs de post-flambage permet de traiter les transitions entre équilibres, qui ont en général lieu en régime post-critique. Des transitions sous-critiques et sur-critiques sont mises en évidence. Un diagramme de stabilité est établi dans un espace à deux dimensions de paramètres adimentionnels. Ces paramètres sont respectivement proportionnels à l'intensité des deux valeurs principales des contraintes dans le film en compression biaxiale et font également intervenir les modules élastiques. Les résultats du calcul sont confrontés aux résultats expérimentaux obtenus avec des films minces de nickel déposés sur substrat de polycarbonate. L'expérience s'appuie sur un dispositif d'observation in situ par microscopie à force atomique (AFM), couplé avec une machine de compression uniaxiale. Ce dispositif 
permet de faire varier l'état de contraintes biaxiales présent dans le film, par compression du substrat (Coupeau et al., 1999).

\section{Procédure expérimentale et motifs de flambage observés}

Les détails du protocole expérimental peuvent êtres trouvés dans (Parry et al., 2004). Les films minces de nickel sont déposés sur des substrats de polycarbonate à température ambiante par pulvérisation ionique. Les épaisseurs de ces films sont comprises entre quelques dizaines et quelques centaines de nanomètres, et sont le siège de fortes contraintes de compression (de l'ordre du GPa) dues à la méthode de déposition. Dans la discussion ultérieure, le film est considéré disposé dans le plan $(O ; x, y)$.

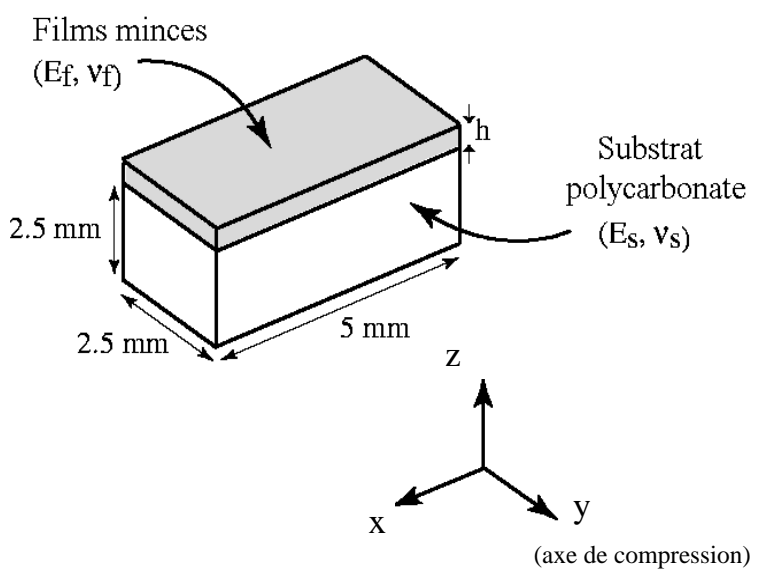

Figure 1. Configuration de l'échantillon et choix des axes

Dans un premier temps, le substrat est comprimé le long de la direction $(O y)$ de manière à induire le délaminage. On voit alors apparaître des bandes de délaminage rectangulaires régulièrement espacées. Ces bandes, dont la longueur est très grande devant la largeur s'étendent dans la direction $(O x)$ et prennent la forme de rides droites dès leur formation (voir la figure 1 pour la configuration des axes et la figure 3 3 a) pour l'image AFM d'une ride droite). Les directions $(O x)$ et $(O y)$ sont appelées respectivement direction longitudinale et direction transversale dans la suite. L'évolution de ces rides droites a été étudiée expérimentalement à l'aide d'un dispositif composé d'un microscope à force atomique, permettant l'observation in situ du film, couplé à une machine de compression. Lors de la compression selon l'axe $(O y)$, les variations des valeurs principales des contraintes de compression dans le film mince sont les suivantes : 


$$
\begin{aligned}
\Delta \sigma_{x x} & =\frac{E_{f}}{E_{s}} \frac{\nu_{f}-\nu_{s}}{1-\nu_{f}^{2}} \sigma_{e x t} \\
\Delta \sigma_{y y} & =\frac{E_{f}}{E_{s}} \frac{1-\nu_{s} \nu_{f}}{1-\nu_{f}^{2}} \sigma_{e x t}
\end{aligned}
$$

où $E_{i}$ et $\nu_{i}$ sont respectivement le module d'Young et le coefficient de Poisson pour le matériau (i), $\sigma_{\text {ext }}$ est la valeur absolue de la contrainte de compression latérale appliquée au substrat de polycarbonate au cours de l'expérience. La contrainte totale de compression $\sigma$ présente dans le film est la somme de la contrainte interne $\sigma_{0}$ liée au procédé de fabrication et de la variation de compression $\Delta \sigma=\left(\Delta \sigma_{x x}, \Delta \sigma_{y y}\right)$. Puisque $\nu_{f}<\nu_{s}$, l'application de la compression supplémentaire provoque dans le film un surcroît de compression dans la direction $(O y)$ et une diminution de la compression dans la direction $(O x)$.

Dans un deuxième temps, la compression extérieure $\sigma_{e x t}$ est ramenée progressivement à zéro. Les rides droites se transforment alors en bulles ou en cordons de téléphone suivant les situations (figure 3 ).

\section{Modèle éléments finis pour les bandes délaminées}

Les parties délaminées du film mince sont modélisées comme des bandes élastiques rectangulaires en contact unilatéral avec un support rigide plan. L'épaisseur de la plaque est notée $h$ et sa longueur est supposée infiniment grande devant sa largeur $b$. Nous nous intéressons aux états d'équilibre présentant une périodicité spatiale, conformément aux observations expérimentales. Le modèle peut alors être réduit au motif élémentaire de la structure périodique. Nous considérons ainsi une plaque rectangulaire de dimensions $b$ et $a$, où $a$ est la période spatiale. La progression du délaminage après la formation de la bande de largeur $b$ n'est pas considérée dans ce problème. Aucune évolution de cette largeur n'est d'ailleurs observée expérimentalement durant les différents cycles de compression/relâchement.

La solution doit satisfaire à la condition d'unilatéralité, i.e. positivité du déplacement hors-plan sur tout le domaine et force de réaction positive sur la surface de contact. Le substrat est très épais par rapport au film et impose de fait un déplacement aux frontières de la plaque qui est calculé explicitement. Les deux bords situés le long du front délaminé satisfont à des conditions aux limites (suivant la direction $O x$ ) de type encastrement :

$$
\begin{aligned}
w & =0 \\
\frac{\partial w}{\partial y} & =0
\end{aligned}
$$


Aux deux extrémités (bords parallèles à la direction $O y$ ), les conditions aux limites prennent en compte la périodicité des motifs :

$$
\begin{aligned}
\frac{\partial w}{\partial x} & =0 \\
\frac{\partial^{3} w}{\partial x^{3}} & =0 \quad \text { ou } \quad w=0 \quad \text { si contact }
\end{aligned}
$$

Des déplacements imposés dans le plan $(O ; x, y)$ sont appliqués sur les quatre côtés de la plaque (voir figure 2 pour les détails). Le chargement en déplacement est déduit des contraintes régnant dans la partie adhérente du film $\left(\sigma_{x x}, \sigma_{y y}\right)$. Les calculs par la méthode des éléments finis sont menés à l'aide du logiciel ABAQUS (Hibbit et al., 2003). Les grands déplacements sont gérés et les conditions unilatérales sont prises en compte par l'introduction d'un plan rigide sous le film.

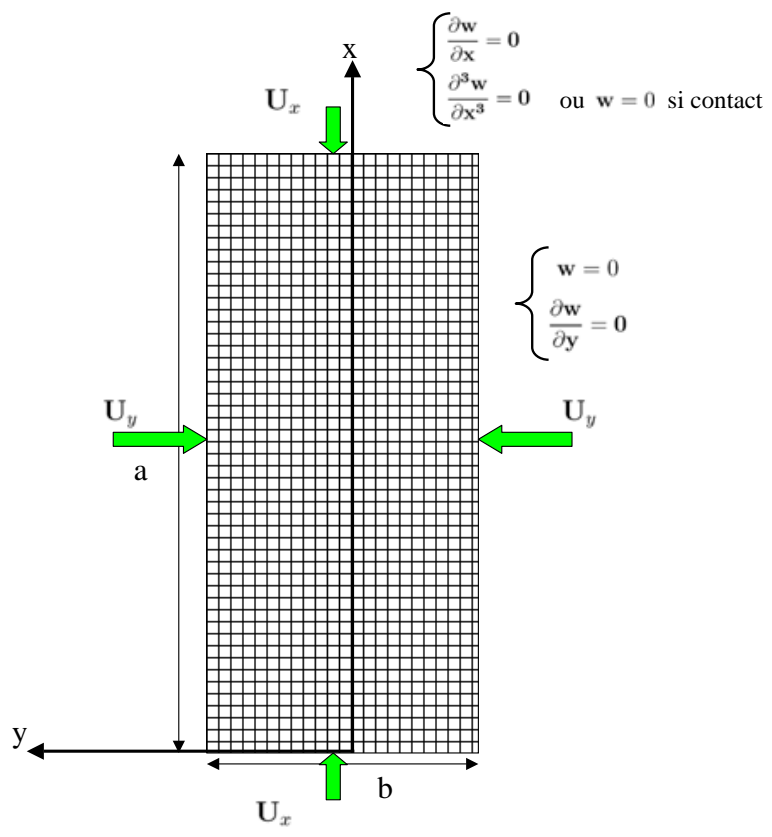

Figure 2. Conditions aux limites sur la plaque rectangulaire modélisant une portion de bande délaminée. Les bords parallèles à la direction $(O x)$, représentant la frontière de la zone délaminée, sont supposés encastrés. Des conditions de symétrie liées à la périodicité des motifs sont retenues pour les bords parallèles à la direction (Oy) 
Le film est maillé par des éléments de coque quadrilatéraux. La procédure standard est utilisée, le calcul est quasi statique. Dans les cas où une transition entre équilibres a un caractère sous-critique, la procédure de Riks est utilisée, comme nous l'expliquons plus loin.

Ainsi, la démarche adoptée dans cette étude est de suivre l'évolution des structures flambées dans leur régime post-critique lointain, sans linéariser autour d'un état d'équilibre particulier et sans introduire de forme a priori pour le mode de flambage. Une attention particulière est portée au respect de la condition d'unilatéralité imposée par le substrat qui a une influence importante sur les modes de flambage. Cette démarche est, à la connaissance des auteurs, originale dans le traitement du flambage des films minces délaminés. Elle a pour but de capter avec précision les phénomènes complexes et fortement non linéaires se déroulant dans le régime post-critique.

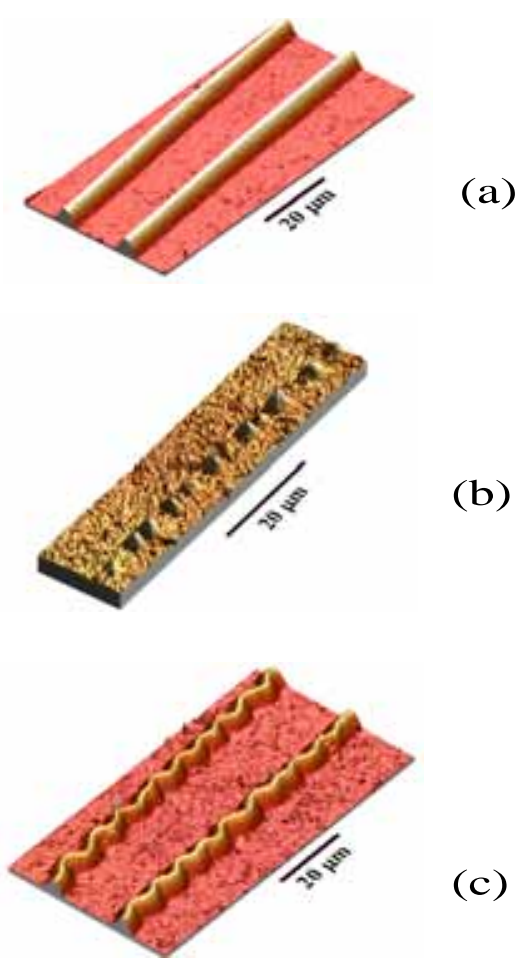

Figure 3. Images AFM des structures de flambage sur film mince de nickel (Coupeau et al.) : (a) rides droites formées perpendiculairement à l'axe de compression. (b) rangées de bulles provenant du flambage des rides droites. (c) structures en cordons de téléphone provenant du flambage des rides droites 


\section{Transitions vers le flambage secondaire}

\subsection{De la ride droite à la distribution de bulles}

La transition intervenant lors de la transformation d'une ride droite en une distribution de bulles a été étudiée (Parry et al., 2004; Parry et al., 2005). Cette transition a lieu lors de l'expérience lorsque la compression extérieure est progressivement relâchée. On note $\rho_{c}$ la valeur critique du rapport $\rho=\sigma_{x x} / \sigma_{y y}$ pour laquelle la transition a lieu ( $\rho$ augmente alors, voir équation [1]).

L'énergie par unité de longueur $e(a)$ de la structure périodique de bulles est calculée à mesure que $\rho$ augmente. La période $a^{*}(\rho)$ minimisant $e$ est identifiée pour chaque valeur de $\rho$.

Pour le flambage naissant ( $\rho$ légérement supérieur à $\rho_{c}$ ), les petites valeurs du rapport $a / b$ sont énergétiquement favorables $(a / b<1)$. Le flambage naissant est caractérisé par une légère ondulation au sommet de la ride droite (figure 44(a)).

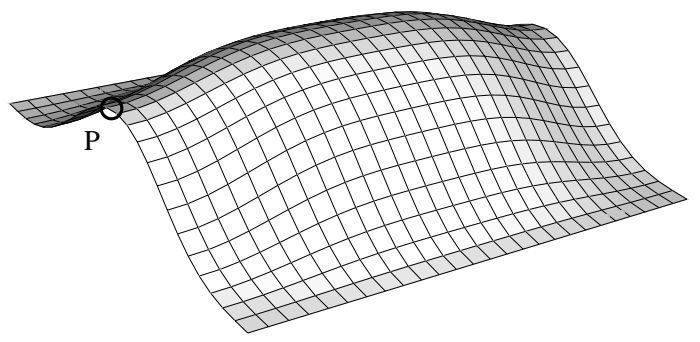

(a)

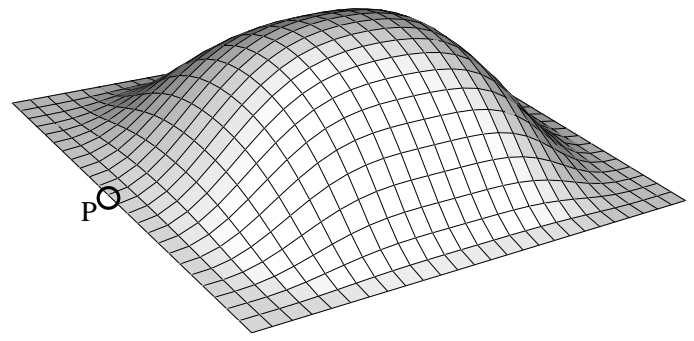

(b)

Figure 4. Calcul par éléments finis des différentes étapes de la transition ride-bulles : (a) flambage naissant, (b) état post-critique lointain avec bords de la bulle déposés sur le substrat. Le déplacement vertical du point $P$ est suivi au cours du calcul 
Mais lorsque $\rho$ continue d'augmenter (i.e. dans le régime post-critique de l'équilibre de bulles), les rapports $a / b$ de valeurs plus grandes réalisent alors le minimum de $e(a)$, autour de 1,2. A ce stade, les bulles deviennent de plus en plus profondes, les parties du film à la périphérie des bulles et les portions de film entre les bulles sont redéposées sur le substrat (figure 4-(b)).

Ces résultats expliquent pourquoi les longueurs de bulles observées expérimentalement sont telles que le rapport $a / b$ est compris entre 1,1 et 1,2 , ce qui est plus grand que le rapport prévu par les résultats de l'analyse du flambage naissant.

On pourrait donc s'attendre à une variation de la longueur des bulles au cours du chargement (i.e. une forte dépendance $a(\rho)$ de la longueur par rapport à l'état de chargement). Du point de vue expérimental, un tel phénomène n'est pas observé. Le calcul permet de constater que la plage de valeurs de $\rho$ pour lesquelles les bulles courtes sont favorables est en réalité assez étroite.

Ainsi, dans l'étude présentée ci-après, qui concerne l'existence d'un rapport $a / b$ critique au-dessus duquel la transition ride-bulles devient sous-critique, la longueur de bulle $a$ est considérée constante au cours de la transition. Les rapports $a / b$ utilisés dans les calculs ont été choisis en conformité avec les observations expérimentales.

Ces calculs ont été menés en s'appuyant sur une procédure de Riks (contrôle en longueur d'arc) de manière à pouvoir passer les points limites. La convergence est difficile à atteindre, même avec cette méthode, et les calculs consomment un temps assez important au regard du nombre relativement limité de noeuds utilisés dans ce modèle. La méthode asymptotique numérique (MAN) (Vannucci et al., 1988; Lahmam et al., 2002) serait sans doute une bonne alternative pour ce problème, mais elle n'est pas implémentée dans ABAQUS pour le moment.

Le phénomène est illustré figure 5 où la hauteur $w$ du point $P$, représenté figure 4 est reportée en fonction de $\rho$. La flèche $w$ a été rendue adimensionelle par division par la flèche maximale de la ride. Pour $\rho=0$, la structure est sous forme de ride droite. L'augmentation de $\rho$ correspond à une diminution de la compression transversale $\sigma_{y y}$ relativement à la compression longitudinale $\sigma_{x x}$. A mesure que la bulle prend forme, le point $P$ descend vers le substrat. Le contact avec le substrat devient effectif pour $w=0$. La bulle est alors formée (i.e. la limite de la zone de contact est un contour fermé, d'aspect elliptique).

Pour $a / b<1,15$, la transition est sur-critique. La hauteur $w$ décroît progressivement à mesure que $\rho$ augmente et la correspondance bi-univoque entre $w$ est $\rho$ reste vraie sur $[0,1]$. Pour $a / b>1,15$, la transition est sous-critique et la forme de la structure change brutalement. L'exemple mis en évidence figure 5 est celui de la formation par claquage d'une bulle de rapport $a / b=1,2$. Dans un premier temps, le chargement est tel que $\rho$ augmente de 0 à 1 . Entre 0 et $\rho_{1}$, le flambage naissant sous forme de bulle s'amorce progressivement, et les ondulations restent petites par rapport à la hauteur de la ride. Pour $\rho=\rho_{1}$, la ride s'affaisse brutalement et la hauteur du point $P$ chute de $A$ à $B$. A la suite de ce claquage, l'augmentation de $\rho$ s'accompagne d'une descente progressive du point $P$ vers le substrat, qui est atteint pour $w=0$. Dans un 
deuxième temps, le chargement est tel que $\rho$ décroît de 1 à 0 . Entre $\rho=1$ et $\rho=\rho_{2}$, les deux bords libres de se déplacer verticalement quittent alors progressivement le niveau du substrat pour reprendre de la hauteur. Lorsque $\rho$ atteint la valeur $\rho_{2}$ la hauteur augmente brutalement et la structure se retrouve de nouveau à l'état de ride droite, la hauteur du point $P$ passant de $A^{\prime}$ à $B^{\prime}$. On peut noter l'hystérèse qui accompagne ce phénomène. On notera que le claquage ne se produit pas pour la même valeur de $\rho$ selon que la structure est en charge ou en décharge $\left(\rho_{1} \neq \rho_{2}\right)$.

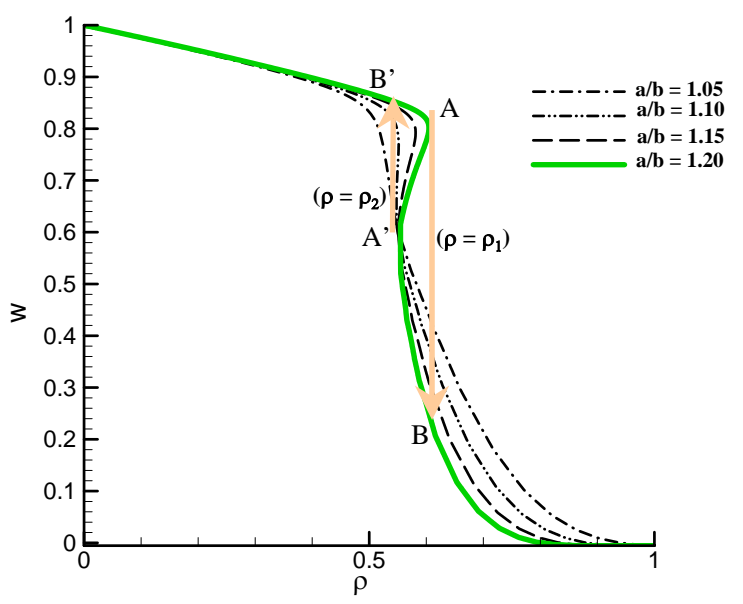

Figure 5. Le déplacement vertical (adimensionnalisé) d'un point situé au milieu d'un bord transversal est tracé en fonction du paramètre de chargement $\rho$ pour différents rapports $a / b$. Le claquage est mis en évidence pour $a / b=1,2$. Il intervient aux points $A$ ( $\rho$ croissant $)$ et $A^{\prime}$ ( $\rho$ décroissant)

La procédure de Riks a été utilisée pour suivre le trajet de chargement représenté par la portion de courbe comprise entre $\rho_{1}$ et $\rho_{2}$. Il s'agit d'une méthode de contrôle en longueur d'arc implémentée dans ABAQUS.

Le phénomène de claquage prédit par ce modèle a été observé expérimentalement lors des phases de chargement-déchargement successives des échantillons de films minces de nickel sur substrat polycarbonate (Parry et al., 2005).

\subsection{De la ride droite au cordon de téléphone}

Le flambage des films minces sous forme de cordons de téléphone est assez souvent observé lors de l'élaboration de films, que ces décollements soient spontanés ou bien provoqués par des actions extérieures. On peut citer quelques exemples d'observations de cordons dans la littérature. Thouless et al. étudient des cordons formés sur un film de silicium déposé sur un substrat $\mathrm{SiO}_{2}$ (Thouless, 1993). L'exemple de films de $\mathrm{SiO}_{2}$ déposés sur substrat de verre ou de nickel est présenté dans (Priest et 
al., 1992), tandis que celui de cordons sur un film de mica déposé sur aluminium est étudié dans (Hutchinson et al., 1992b).

Les cordons de téléphone proviennent du flambage de rides droites devenues instables sous fortes contraintes, comme l'a montré l'étude de la perturbation de la ride droite (Audoly, 1999; Cleymand et al., 2001; Moon et al., 2004).

L'expérience de compression d'un film de nickel sur polycarbonate décrite précédemment permet de visualiser la transition ride-cordons. Rappelons que la compression extérieure, qui agit perpendiculairement aux bandes délaminées, tend à surcomprimer celles-ci latéralement. Dans ce contexte l'équilibre de ride droite est favorable. Lorsque la compression extérieure est relâchée, le taux de compression axial $\left(\frac{\sigma_{x x}}{\sigma_{y y}}\right)$ des bandes délaminées augmente et l'équilibre en cordons de téléphone devient plus stable que la ride droite. Une transition ride-cordon s'amorce alors. Des vues AFM montrant cette transition ont été réalisées figure 6
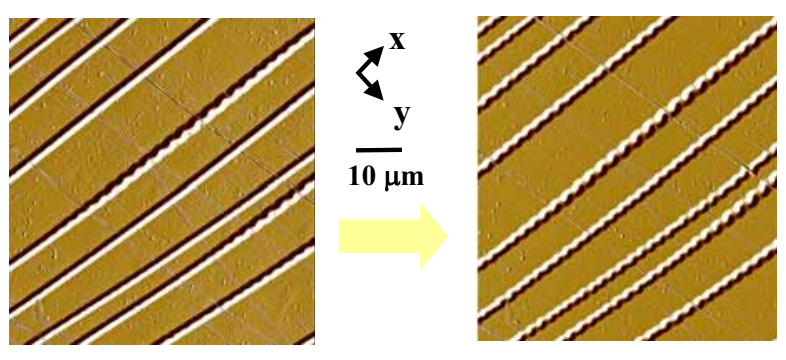

Figure 6. Observations par microscopie à force atomique du flambage des rides droites sous forme de cordons de téléphone. Le flambage apparaît localement puis s'étend le long des rides

On constate que le flambage sous forme de cordons de téléphone se produit d'abord localement dans certaines zones sur lesquelles on voit apparaitre quelques ondulations. Puis les ondulations se propagent le long des rides droites.

Enfin, lorsque bulles et cordons coexistent sur le même échantillon, l'observation montre que les cordons se développent sur des bandes de plus grande largeur. On constate le même comportement lors du calcul par éléments finis en se plaçant dans les conditions de chargement expérimentales et en augmentant la largeur $b$ des bandes.

On modélise la naissance du flambage sous forme de cordons de téléphone par éléments finis. Pour cela, on choisit une portion de bande délaminée assez longue $(a=8 b)$. Une légère imperfection est introduite à l'une des extrémités de manière à amorcer le phénomène. Les conditions aux limites sont les mêmes que celles employées dans le cas de l'étude de la transition ride-bulles étudiée précédemment, i.e. encastrement le long des bords délaminés et conditions de raccord périodique aux extrémités de la bande. Le chargement subi par la bande élastique lors de l'expérience est traduit dans la modélisation numérique en termes de déplacements imposés à la 
frontière. On peut distinguer deux phases de calcul, correspondant à l'étape de chargement extérieur et à l'étape de relaxation du chargement extérieur. Pendant la première phase, la solution obtenue est la ride droite. Durant la deuxième phase, pendant laquelle la compression latérale diminue et la compression axiale augmente légèrement, on observe d'abord une diminution de la hauteur de la ride, jusqu'à une valeur critique du chargement. Alors, des ondulations commencent à apparaître sur la ride au niveau du défaut pour cette valeur critique du chargement. Elles se propagent ensuite le long du cordon pour de petites variations du chargement à partir du défaut (figure 7). Le flambage local provoque une réorganisation des contraintes de proche en proche qui permet au cordon de s'étendre progressivement le long de la ride. On constate que le flambage sous forme de cordons de téléphone est sur-critique (flambage sous force croissante) contrairement au flambage sous forme de bulles, qui peut être sous-critique à partir d'une certaine taille de bulle, comme vu précédemment.
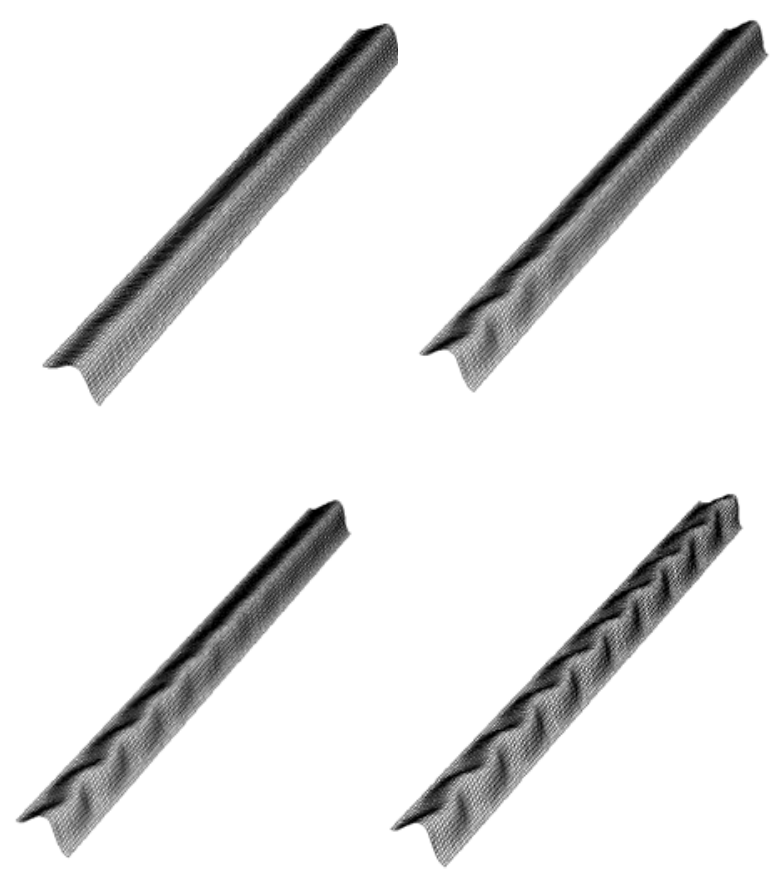

Figure 7. Calcul par éléments finis du flambage d'une ride droite sous forme de cordon de téléphone à partir d'un défaut ponctuel introduit à une extrémité. La région flambée s'étend progressivement le long de la structure 


\section{Diagramme de stabilité pour les modes de flambage}

En appliquant des conditions aux limites idoines sur les frontières du domaine situées perpendiculairement à $O x$ (en travers de la bande délaminée), on peut représenter les modes de flambage périodiques dont la période est un diviseur de $a$. Les bords de la zone délaminée $y=0$ et $y=b$ sont encastrés. Les frontières sont chargées en déplacement $(U x, U y)$. Les conditions de chargement seront cependant exprimées en fonction de la contrainte biaxiale $\left(\bar{\sigma}_{x x}, \bar{\sigma}_{y y}\right)$ s'exercant dans la partie adhérente du film.

De précédentes études ont mis en évidence l'influence du rapport $\frac{\sigma_{x x}}{\sigma_{y y}}$ sur la stabilité des modes de flambage en rides droites, bulles et cordons ((Audoly, 1999; George et al., 2002)). Un diagramme de stabilité dans le plan $\sigma_{y y}, \sigma_{x x}$ a été obtenu fournissant des informations intéressantes sur la stabilité des différents équilibres (Audoly et al., 2002). Cependant la résolution est approximative, d'une part parce que les auteurs émettent a priori une hypothèse sur la forme des solutions et d'autre part parce qu'ils ne prennent pas en compte le contact entre le film et le support. Ce contact se révèle cependant significatif pour de forts niveaux de contraintes. L'objectif de notre étude est de prendre en compte ces effets dans la construction de la cartographie des équilibres stables.

Les paramètres pertinents à considerer pour obtenir une cartographie indépendante des dimensions du film et des caractéristiques mécaniques, sont les paramètres adimensionels suivants $\left(\frac{\bar{\sigma}_{y y}}{\bar{E}} \frac{b}{h}, \frac{\bar{\sigma}_{x x}}{\bar{E}} \frac{b}{h}\right)$ avec $\bar{E}=\frac{E}{1-\nu^{2}}$. On notera par la suite $\left(\beta_{t}, \beta_{l}\right)$ ces paramètres ( $t$ pour transversal et $l$ pour longitudinal). Le calcul a mis en évidence quatre types de solutions qui sont l'état plan, la ride droite, le cordon de téléphone et la rangée de bulles.

L'idée de la cartographie est de déterminer l'équilibre le plus stable pour chaque point de l'espace $\left(\beta_{t}, \beta_{l}\right)$, et à cette fin de déterminer les frontières séparant les différentes zones de stabilité. Pour ce faire, on choisit un point de destination $\left(\beta_{t}^{f}, \beta_{l}^{f}\right)$. Puis un chemin dans l'espace $\left(\beta_{t}, \beta_{l}\right)$ qui détermine la manière de faire passer le chargement de $(0,0)$ à $\left(\beta_{t}^{f}, \beta_{l}^{f}\right)$. Soit $\left\{\left(\beta_{t}(\tau), \beta_{l}(\tau)\right), \tau \in[0,1]\right\}$ ce chemin. Pour un état de chargement donné $\left(\beta_{t}, \beta_{l}\right)$, soit $m$ le nombre d'équilibres candidats, $1<m \leq 4$. Un équilibre particulier $k \in\{1 . . m\}$ est caractérisé par l'énergie de déformation $E^{k}\left(\beta_{t}, \beta_{l}, a\right)$, et l'énergie de déformation par unité de longueur est $e^{k}=E^{k} / a$. Dans le cas d'une ride droite, qui est une structure invariante par translation suivant $O x, a$ peut être choisi arbitrairement. Pour les structures périodiques (bulles et cordons), la longueur d'onde $\lambda^{k}\left(\beta_{t}, \beta_{l}\right)$ est choisie en minimisant l'énergie de déformation par unité de longueur et par rapport à $a$. L'équilibre stable dans une condition de chargement est celui qui réalise le minimum $e^{* i}\left(\beta_{t}(\tau), \beta_{l}(\tau)\right)=e^{* i}(\tau)$ de l'énergie $\left\{e^{k}\left(\beta_{t}, \beta_{l}, \lambda^{k}\left(\beta_{t}, \beta_{l}\right)\right) k \in\{1 . . m\}\right\}$. Il est ainsi possible de suivre l'évolution de $e^{* i}(\tau)$ au fur et à mesure du chargement. Une frontière est traversée lorsque, pour $i \neq j$, on a $e^{* i}(\tau)=e^{* j}(\tau)$. Le diagramme de stabilité obtenu est reporté figure 8 . La cartographie obtenue est qualitativement semblable à celle de (Audoly et al., 2002), 
au niveau de l'allure globale du diagramme. Cependant les différences quantitatives sont notables.

Dans (Audoly, 1999), l'auteur discute d'une valeur critique du coefficient de Poisson pour expliquer la présence de bulles ou bien de cordons de téléphone sur un film. La transition se ferait d'une ride vers un cordon de téléphone pour les valeurs du coefficient de Poisson au-delà de la valeur critique, alors qu'une transition d'une ride vers une rangée de bulles serait observée en deçà de la valeur critique. Cela dit, cette interprétation ne permet pas d'expliquer la coexistence de bulles et de cordons de téléphone que l'on peut observer sur un même échantillon. Ce diagramme permet quant à lui de fournir une explication aux coexistences de bulles et de cordons. En effet, on constate que les cordons apparaissent sur des bandes délaminées plus larges que les bulles (respectivement $6 \mu \mathrm{m}$ et $4 \mu \mathrm{m}$ ). On reporte sur le diagramme (figure 8 ) les deux chargements expérimentaux où (1) correspond à la compression extérieure maximale et (2) est atteint lorsque la compression extérieure est relâchée. On a bien confirmation de l'influence de la largeur des bandes délaminées sur la nature de l'équilibre.

\section{Conclusion}

La modélisation par éléments finis du flambage et du post-flambage unilatéral des films minces déposés sur substrat permet de mieux appréhender les différents phénomènes observés expérimentalement. Les résultats présentés concernent le comportement de bandes parallèles sous contrainte de compression biaxiale. Ces bandes sont désolidarisées de leur support en leur faisant subir une surcompression transversale qui provoque une première bifurcation en forme de rides droites. Lors de la relaxion de la surcompression latérale, la contrainte longitudinale augmente et induit une seconde bifurcation conduisant à la formation de bulles ou de cordons de téléphone, suivant les valeurs des paramètres.

La longueur d'onde optimale des structures se modifie au cours de l'évolution post-critique. Celle des bulles observées dans l'expérience a été retrouvée. L'existence d'une valeur critique de l'aspect de forme des bulles (longueur/largeur) au-delà de laquelle la bifurcation change de nature, passant de sur-critique à sous-critique et donnant lieu à un claquage a été mise en évidence, conformément aux observations expérimentales. Une cartographie de la stabilité des états d'équilibres a été fournie dans un espace à deux paramètres adimensionnels, caractéristiques de l'état d'équilibre devant se réaliser. L'existence de frontières à l'intérieur de cet espace de paramètres permet d'expliquer la coexistence de rides et de bulles ou de rides et de cordons. 
954 REMN - 16/2007. Modélisations numériques en mécanique

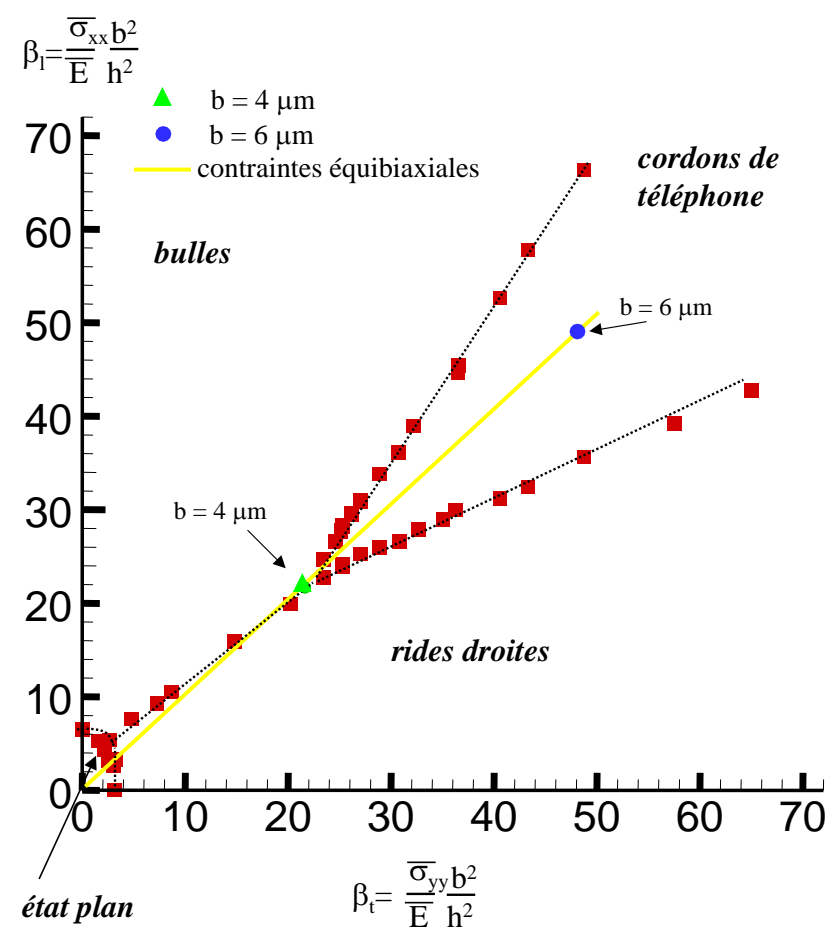

Figure 8. Diagramme de stabilité

\section{Bibliographie}

Audoly B., « Stability of Straight Delamination Blisters », Physical Review Letters, vol. 83, $n^{\circ} 20$, p. 4124, 1999.

Audoly B., Roman B., Pocheau A., « Secondary buckling patterns of a thin plate under in-plane compression », European Physical Journal B., vol. 27, p. 7, 2002.

Cleymand F., Coupeau C., Grilhé J., « Experimental investigation of the instability of buckling patterns : from straight-sided to wormlike structures », Scripta Materialia, vol. 44, p. 2623, 2001.

Coupeau C., Naud J.-F., Cleymand F., Goudeau J., Grilhé J., « Atomic force microscopy of in situ deformed nickel thin films », Thin Solid Films, vol. 353, p. 194, 1999.

George M., Coupeau C., Colin J., Cleymand F., Grilhé J., « Delamination of metal thin films on polymer substrates : from straight-sided blisters to varicose structures », Philosophical Magazine A, vol. 82, p. 633, 2002.

Gille G., Rau B., « Buckling instability and adhesion of carbon layers », Thin Solid Films, vol. 120, p. 109, 1984. 
Gioia G., Ortiz M., « Determination of thin-film debonding parameters from telephone-cord measurements », Acta Materialia, vol. 46, p. 169, 1997.

Hibbit, Karlsson, Sorensen, ABAQUS version 6.3 user's manual. 2003.

Hutchinson J., « Delamination of compressed films on curved substrates », Journal of the Mechanics and Physics of Solids, vol. 49, p. 1847, 2001.

Hutchinson J., Suo Z., « Mixed mode cracking in layered materials », Advances in Applied Mechanics, vol. 29, p. 63, 1992a.

Hutchinson J., Thouless M., Liniger E., « Growth and Configurational Stability of Circular, Buckling-Driven Film Delaminations », Acta Metallica Materiallia, vol. 40, p. 295, 1992b.

Lahmam H., Cadou J.-M., Zahrouni H., Damil N., Potier-Ferry M., « High-order predictorcorrector algorithms », International Journal of Numerical Methods in Engineering, vol. 55, p. 685, 2002.

Moon M.-W., Lee K.-R., Oh K., Hutchinson J., « Buckle delamination on patterned substrates », Acta Materialia, vol. 52, p. 3151, 2004.

Parry G., Colin J., Coupeau C., Foucher F., Cimetière A., Grilhé J., « Snap-through occuring in the post-buckling of thin films », Applied Physics Letters, vol. 86, p. 081905, 2005.

Parry G., Coupeau C., Colin J., Cimetière A., Grilhé J., « Buckling and post-buckling of stresses straight-sided wrinkles : experimental AFM observations of bubbles formation and finite element simulations », Acta Marerialia, vol. 52, p. 3959, 2004.

Priest J., Caswell H.L.and Budo Y., Transactions of the Ninth National Symposium of the American Vacuum Society, McMillan, New York, 1992.

Thouless M., « Combined buckling and cracking of films », Journal of the American Ceramic Society, vol. 76, p. 2936, 1993.

Vannucci P., Cochelin B., Damil N., Potier-Ferry M., « An asymptotic-numerical method to compute bifurcating branches », International Journal of Numerical Methods in Engineering, vol. 41, p. 1365, 1988.

Wang J., Evans A., « Effects of strain cycling on buckling, cracking, and spalling of a thermally grown alumina on a nickel-based bond coat », Acta Materiallia, vol. 47, p. 699, 1999. 